Genet. Sel. Evol. 30 (1998) 89-101

(C) Inra/Elsevier, Paris

Original article

\title{
A simple method of computing restricted best linear unbiased prediction of breeding values
}

\author{
Masahiro Satoh \\ Department of Animal Breeding and Genetics, \\ National Institute of Animal Industry, P.O. Box 5, \\ Tsukuba Norin-kenkyudanchi, Tsukuba-shi 305, Japan
}

(Received 6 May 1997; accepted 12 February 1998)

\begin{abstract}
Restricted best linear unbiased prediction (restricted BLUP) is derived by imposing restrictions directly within a multiple trait mixed model. As a result, the restricted BLUP procedure requires the solution of high order simultaneous equations. In the present paper, a simple method for computing restricted BLUP of breeding values is presented. The technique is valuable, particularly when a large number of restrictions are imposed in a multiple trait mixed model such as constraints of achieving predetermined relative rates of genetic improvement for all traits. (C) Inra/Elsevier, Paris
\end{abstract}

mixed model method / restricted BLUP

Résumé - Méthode simple de calcul d'un BLUP restreint. Le BLUP restreint est calculé directement en posant les contraintes en sus des équations correspondantes à un modèle linéaire mixte multivariate. En conséquence, la procédure du BLUP restreint demande la résolution d'un plus grand nombre d'équations que dans le cas habituel. Dans cet article, on présente un reparamétrage qui permet d'aboutir à un système plus simple et de taille réduite. Cette technique est particulièrement intéressante quand un grand nombre de restrictions est imposé dans un modèle mixte multivariate, comme quand on cherche à obtenir des rapport prédéterminés de progrès génétiques pour tous les caractères.

(c) Inra/Elsevier, Paris

modèle mixte multivariate / BLUP restreint

\section{INTRODUCTION}

Kempthorne and Nordskog [9] gave the basic derivation of restricted selection indices. The model assumed was that the observation vector $\mathbf{y}$, is $\mathbf{y}=\mathbf{f}+\mathbf{u}+\mathbf{e}, \mathbf{u}$ and $\mathbf{e}$ are multivariates normally distributed with $E(\mathbf{u})=E(\mathbf{e})=\mathbf{0}$, and $\mathbf{f}$ is fixed and assumed known. $\operatorname{Var}(\mathbf{u})=\mathbf{I} \otimes \mathbf{G}_{0}, \operatorname{var}(\mathbf{e})=\mathbf{I} \otimes \mathbf{R}_{0}$, and $\operatorname{cov}\left(\mathbf{u}, \mathbf{e}^{\prime}\right)=\mathbf{0}$, where

E-mail: hereford@niai.affrc.go.jp 
$\mathbf{G}_{0}$ is the genetic variance-covariance matrix, $\mathbf{R}_{0}$ is the environmental variancecovariance matrix, and $\otimes$ is the direct product operation. They were interested in maximizing improvement in $\mathbf{m}^{\prime} \mathbf{u}_{i}$, but at the same time not altering the expected $\mathbf{C}_{0}^{\prime} \mathbf{u}_{i}$, in the candidate for selection, where $\mathbf{m}$ is a vector of relative weights, $\mathbf{u}_{i}$ is the subvector of $\mathbf{u}$ pertaining to the $i$ th animal, and $\mathbf{C}^{\prime} 0$ has $r$ linearly independent rows. They proved that such a restricted selection index is $\mathbf{b}^{\prime} \mathbf{y}_{i}$, where $\mathbf{y}_{i}$ is the subvector of $\mathbf{y}$ pertaining to the $i$ th animal, and $\mathbf{b}$ is the solution to

$$
\left[\begin{array}{cc}
\mathbf{G}_{0}+\mathbf{R}_{0} & \mathbf{G}_{0} \mathbf{C}_{0} \\
\mathbf{C}_{0}^{\prime} \mathbf{G}_{0} & \mathbf{0}
\end{array}\right]\left[\begin{array}{l}
\mathbf{b} \\
\boldsymbol{\theta}
\end{array}\right]=\left[\begin{array}{c}
\mathbf{G}_{0} \mathbf{m} \\
\mathbf{0}
\end{array}\right]
$$

Index theory was further extended to include various restrictions by Mallard [11], Harville [3, 4], among others. In practical applications, however, large data sets with unknown means and related animals render restricted selection index predictors of breeding values impossible to compute. Quaas and Henderson [13, 14] extended the BLUP procedure of Henderson [5] to allow estimation of breeding values including restrictions for no genetic change among correlated traits (restricted BLUP).

Restricted BLUP was derived by imposing restrictions directly on the multiple trait mixed model equations. Consequently, the restricted BLUP procedure requires solutions of high order simultaneous equations, particularly when a large number of animals are evaluated for many traits. For this reason, computational techniques have been studied for computing restricted BLUP. Lin [10] showed how restricted BLUP of breeding values can be estimated not only for zero change but also for proportional change in restricted traits. It was, however, assumed that the variancecovariance matrix among predicted breeding values was the same as that among true breeding values. This approach adds bias when estimates of genetic variances and covariances are used instead of the true parameters. The assumption ignores the effect of differing accuracies of prediction of individual breeding values for each animal, particularly when animal models are fitted to large unbalanced field data sets [15]. Itoh and Iwaisaki [7] found that a canonical transformation of the traits to new independent variables was possible and, consequently, only mixed model equations of relatively smaller order for each trait need to be solved. However, this is applicable only to an animal model with identical models for all traits and no partially missing observations.

The objectives of the present paper are to show a simple procedure for computing restricted BLUP of breeding values and to discuss its application.

\section{THEORETICAL APPROACH}

\subsection{Theoretical background}

An additive genetic mixed animal model for $q$ traits is assumed. The model for the $i$ th trait is written as:

$$
\mathbf{y}_{i}=\mathbf{X}_{i} \mathbf{b}_{i}+\mathbf{Z}_{i} \mathbf{u}_{i}+\mathbf{e}_{i}
$$

where $\mathbf{y}_{i}$ is a vector of observations for the $i$ th trait; $\mathbf{b}_{i}$ is a vector of unknown fixed effects; $\mathbf{X}_{i}$ is a known incidence matrix relating elements of $\mathbf{b}_{i}$ to $\mathbf{y}_{i}, \mathbf{u}_{i}$ is a 
vector of unknown random additive genetic effects, $\mathbf{Z}_{i}$ is a known incidence matrix relating elements of $\mathbf{u}_{i}$ to $\mathbf{y}_{i}$, and $\mathbf{e}_{i}$ is a vector of random errors. Let $n_{j}$ be the number of records on the $j$ th animal; $j=1,2, \ldots, n$ and $0 \leqslant n_{j} \leqslant q$. The model for all traits is written as:

$$
\mathbf{y}=\mathbf{X b}+\mathbf{Z u}+\mathbf{e}
$$

where records are ordered by animals within traits. It is assumed that $\mathbf{u}$ and $\mathbf{e}$ are multivariates normally distributed with $E(\mathbf{u})=\mathbf{0}, E(\mathbf{e})=\mathbf{v} 0, \operatorname{var}(\mathbf{u})=\mathbf{G}$, $\operatorname{var}(\mathbf{e})=\mathbf{R}$, and $\operatorname{cov}\left(\mathbf{u}, \mathbf{e}^{\prime}\right)=\mathbf{0} ; \mathbf{G}=\mathbf{G}_{0} \otimes \mathbf{A}$, where $\mathbf{G}_{0}$ is a $q \times q$ additive genetic variance-covariance matrix for the $q$ traits, $\mathbf{A}$ is the additive relationship matrix for the $n$ animals, and $\mathbf{R}$ is an $n \times n\left(n=\Sigma n_{i}\right)$ error variance-covariance matrix for the $q$ traits for the $n$ animals.

Let the set of restrictions on $\mathbf{u}$ be $\mathbf{C}^{\prime} \mathbf{u}$. If the same constraints are imposed on the additive genetic values of all animals, $\mathbf{C}=\mathbf{C}_{0} \otimes \mathbf{I}_{m}$ where $\mathbf{C}_{0}$ is a $q \times r$ matrix with full column rank; $m$ is the number of animals represented in $\mathbf{u}$. The number of columns of $\mathbf{C}_{0}, r$, depends on the number and type of constraints imposed: no change and/or proportional change. This will be illustrated subsequently. Kempthorne and Nordskog [9] defined $\mathbf{C}_{0}$ for no change constraints. For example, if the restriction is no change for the first two traits, $\mathbf{C}_{0}$ might be

$$
\mathbf{C}_{0}^{\prime}=\left[\begin{array}{lllll}
1 & 0 & 0 & \ldots & 0 \\
0 & 1 & 0 & \ldots & 0
\end{array}\right]
$$

Here $r$ is the number of traits constrained. For the case of proportional constraints (involving $2 \leqslant p \leqslant q$ traits), define:

$$
\mathbf{C}_{0}^{\prime}=\left[\begin{array}{ccccc}
c_{p} & 0 & \ldots & 0 & -c_{1} \\
0 & c_{p} & \ldots & 0 & -c_{2} \\
\vdots & \vdots & & \vdots & \vdots \\
0 & 0 & \ldots & c_{p} & -c_{p-1}
\end{array}\right]
$$

Then let $\mathbf{C}_{0}^{\prime}=\left[\begin{array}{ll}\mathbf{C}_{p}^{\prime} & \mathbf{0}_{(p-1) \times(q-p)}\end{array}\right]$

where $c_{i}$ is a predetermined proportional change for traits $1,2, \ldots, p[8,11]$. Note that $r=p-1$. Furthermore, if constraints include no change and proportional change, $\mathbf{C}_{0}$ is $z$ columns whose elements are unity or zero in addition to $\mathbf{C}_{p}$, and then $r=z+p-1$. For example, if we want no change in trait 1 but proportional change in traits 2,3 and 4 is desired based on proportional constraints in the ratio $2: 3: 4, \mathbf{C}_{0}$ is expressed as

$$
\mathbf{C}_{0}^{\prime}=\left[\begin{array}{ccccccc}
1 & 0 & 0 & 0 & 0 & \ldots & 0 \\
0 & 4 & 0 & -2 & 0 & \ldots & 0 \\
0 & 0 & 4 & -3 & 0 & \ldots & 0
\end{array}\right]
$$

The restricted BULP of $\mathbf{u}, \hat{\mathbf{u}}$, is obtained by solving the following equations [13]:

$$
\left[\begin{array}{lll}
\mathbf{X}^{\prime} \mathbf{R}^{-1} \mathbf{X} & \mathbf{X}^{\prime} \mathbf{R}^{-1} \mathbf{Z} & \mathbf{X}^{\prime} \mathbf{R}^{-1} \mathbf{Z G C} \\
\mathbf{Z}^{\prime} \mathbf{R}^{-1} \mathbf{X} & \mathbf{Z}^{\prime} \mathbf{R}^{-1} \mathbf{Z}+\mathbf{G}^{-1} & \mathbf{Z}^{\prime} \mathbf{R}^{-1} \mathbf{Z G C} \\
\mathbf{C}^{\prime} \mathbf{G} \mathbf{Z}^{\prime} \mathbf{R}^{-1} \mathbf{X} & \mathbf{C}^{\prime} \mathbf{G} \mathbf{Z}^{\prime} \mathbf{R}^{-1} \mathbf{Z} & \mathbf{C}^{\prime} \mathbf{G} \mathbf{Z}^{\prime} \mathbf{R}^{-1} \mathbf{Z G C}
\end{array}\right]\left[\begin{array}{l}
\mathbf{6} \\
\hat{\mathbf{u}} \\
\hat{\mathbf{w}}
\end{array}\right]=\left[\begin{array}{l}
\mathbf{X}^{\prime} \mathbf{R}^{-1} \mathbf{y} \\
\mathbf{Z}^{\prime} \mathbf{R}^{-1} \mathbf{y} \\
\mathbf{C}^{\prime} \mathbf{G} \mathbf{Z}^{\prime} \mathbf{R}^{-1} \mathbf{y}
\end{array}\right]
$$

where $\mathbf{B}$ is a vector of some solution to $\mathbf{b}$ and $\hat{\mathbf{w}}$ is a vector of Lagrange multipliers. 


\subsection{Restriction for general case}

Premultiplying the second equation in (3) by $\mathbf{C}^{\prime} \mathbf{G}$ and then subtracting from this product the third equation gives

$$
\mathbf{C u}=\mathbf{0}
$$

This implies that some îs are null and/or there are simple linear dependencies among them. These can be exploited to reduce the size of the problem. Now we consider imposing the same restrictions on the predicted breeding values of all animals, however, it is possible to relax the situation. When constraints are imposed on some traits, model (1) can be rewritten as

$$
\left[\begin{array}{l}
\mathbf{y}_{\mathrm{Z}} \\
\mathbf{y}_{\mathrm{R}} \\
\mathbf{y}_{\mathrm{N}}
\end{array}\right]=\left[\begin{array}{ccc}
\mathbf{x}_{\mathrm{Z}} & \mathbf{0} & \mathbf{0} \\
\mathbf{0} & \mathbf{x}_{\mathrm{R}} & \mathbf{0} \\
\mathbf{0} & \mathbf{0} & \mathbf{x}_{\mathrm{N}}
\end{array}\right]\left[\begin{array}{l}
\mathbf{b}_{\mathrm{Z}} \\
\mathbf{b}_{\mathrm{R}} \\
\mathbf{b}_{\mathrm{N}}
\end{array}\right]+\left[\begin{array}{ccc}
\mathbf{z}_{\mathrm{Z}} & \mathbf{0} & \mathbf{0} \\
\mathbf{0} & \mathbf{Z}_{\mathrm{R}} & \mathbf{0} \\
\mathbf{0} & \mathbf{0} & \mathbf{Z}_{\mathbf{n}}
\end{array}\right]\left[\begin{array}{l}
\mathbf{u}_{\mathrm{Z}} \\
\mathbf{u}_{\mathrm{R}} \\
\mathbf{u}_{\mathrm{N}}
\end{array}\right]+\left[\begin{array}{l}
\mathbf{e}_{\mathrm{Z}} \\
\mathbf{e}_{\mathrm{R}} \\
\mathbf{e}_{\mathrm{N}}
\end{array}\right]
$$

where subscripts $\mathrm{Z}, \mathrm{R}$ and $\mathrm{N}$ correspond to $z$ characters with no change, $p$ characters with proportional constraints and $q-z-p$ characters without constraint, respectively. From (4),

$$
\mathbf{C}^{\prime} \hat{\mathbf{u}}=\left(\mathbf{C}_{0}^{\prime} \otimes \mathbf{I}_{m}\right) \hat{\mathbf{u}}=\left[\begin{array}{ccc}
\mathbf{I}_{\mathrm{z}} & \mathbf{0} & \mathbf{0} \\
\mathbf{0} & \mathbf{C}_{p}^{\prime} & \mathbf{0}
\end{array}\right] \otimes \mathbf{I}_{m}\left[\begin{array}{c}
\hat{\mathbf{u}}_{\mathrm{Z}} \\
\hat{\mathbf{u}}_{\mathrm{R}} \\
\hat{\mathbf{u}}_{\mathrm{N}}
\end{array}\right]=\mathbf{0}
$$

Then,

$$
\hat{\mathbf{u}}_{\mathrm{Z}}=\mathbf{0}
$$

and from (2) and (5),

$$
\hat{\mathbf{u}}_{\mathrm{R}}=\mathbf{k} \otimes \hat{\mathbf{u}}_{p}=\left(\mathbf{k} \otimes \mathbf{I}_{m}\right) \hat{\mathbf{u}}_{p}
$$

where $\mathbf{k}^{\prime}=\left[\begin{array}{lllll}\mathrm{c}_{1} / \mathrm{c}_{p} & \mathrm{c}_{2} / \mathrm{c}_{p} & \ldots & \mathrm{c}_{p-1} / \mathrm{c}_{p} & 1\end{array}\right]=\left[\begin{array}{llll}\mathrm{k}_{1} & \mathrm{k}_{2} & \ldots & \mathrm{k}_{p}\end{array}\right]$ and $\hat{\mathbf{u}}_{\mathrm{R}}=\left[\begin{array}{llll}\hat{\mathbf{u}}_{1}^{\prime} & \hat{\mathbf{u}}_{2}^{\prime} & \cdots & \hat{\mathbf{u}}_{p}^{\prime}\end{array}\right]^{\prime}$.

Using (6) and (7),

$$
\hat{\mathbf{u}}=\left[\begin{array}{c}
\hat{\mathbf{u}}_{\mathrm{Z}} \\
\hat{\mathbf{u}}_{\mathrm{R}} \\
\hat{\mathbf{u}}_{\mathrm{N}}
\end{array}\right]=\left[\begin{array}{c}
\mathbf{0} \\
\left(\mathbf{k} \otimes \mathbf{I}_{m}\right) \hat{\mathbf{u}}_{p} \\
\left(\mathbf{I}_{\mathrm{N}} \otimes \mathbf{I}_{m}\right) \hat{\mathbf{u}}_{\mathrm{N}}
\end{array}\right]=\left[\begin{array}{cc}
\mathbf{0} & \mathbf{0} \\
\mathbf{k} & \mathbf{0} \\
\mathbf{0} & \mathbf{I}_{\mathrm{N}}
\end{array}\right] \otimes \mathbf{I}_{\mathrm{m}}\left[\begin{array}{c}
\hat{\mathbf{u}}_{p} \\
\hat{\mathbf{u}}_{\mathrm{N}}
\end{array}\right]=\left(\mathbf{K}_{0} \otimes \mathbf{I}_{m}\right) \tilde{\mathbf{u}}=\mathbf{K} \tilde{\mathbf{u}}
$$

where $\mathbf{K}_{0}^{\prime}=\left[\begin{array}{ccc}\mathbf{0} & \mathbf{k}^{\prime} & \mathbf{0} \\ \mathbf{0} & \mathbf{0} & \mathbf{I}_{\mathrm{N}}\end{array}\right]$ and $\tilde{\mathbf{u}}=\left[\begin{array}{ll}\hat{\mathbf{u}}_{p}^{\prime}, & \hat{\mathbf{u}}_{\mathrm{N}}^{\prime}\end{array}\right]^{\prime}$. Substituting (8) into (3) and premultiplying both sides appropriately to maintain symmetry, we obtain

$\left[\begin{array}{lll}\mathbf{X}^{\prime} \mathbf{R}^{-1} \mathbf{X} & \mathbf{X}^{\prime} \mathbf{R}^{-1} \mathbf{Z K} & \mathbf{X}^{\prime} \mathbf{R}^{-1} \mathbf{Z G C} \\ \mathbf{K}^{\prime} \mathbf{Z}^{\prime} \mathbf{R}^{-1} \mathbf{X} & \mathbf{K}^{\prime} \mathbf{Z}^{\prime} \mathbf{R}^{-1} \mathbf{Z K}+\mathbf{K}^{\prime} \mathbf{G}^{-1} \mathbf{K} & \mathbf{K}^{\prime} \mathbf{Z}^{\prime} \mathbf{R}^{-1} \mathbf{Z G C} \\ \mathbf{C}^{\prime} \mathbf{G} \mathbf{Z}^{\prime} \mathbf{R}^{-1} \mathbf{X} & \mathbf{C}^{\prime} \mathbf{G} \mathbf{Z}^{\prime} \mathbf{R}^{-1} \mathbf{Z K} & \mathbf{C}^{\prime} \mathbf{G} \mathbf{Z}^{\prime} \mathbf{R}^{-1} \mathbf{Z G C}\end{array}\right]\left[\begin{array}{l}\mathbf{G} \\ \tilde{\mathbf{u}} \\ \hat{\mathbf{w}}\end{array}\right]=\left[\begin{array}{l}\mathbf{X}^{\prime} \mathbf{R}^{-1} \mathbf{y} \\ \mathbf{K}^{\prime} \mathbf{Z}^{\prime} \mathbf{R}^{-1} \mathbf{y} \\ \mathbf{C}^{\prime} \mathbf{G Z}^{\prime} \mathbf{R}^{-1} \mathbf{y}\end{array}\right]$ 
There are fewer ûs in (9) than ûs in (3). Equations (9) show that computation for formulating BLUP equations is relatively simpler than (3).

\subsection{Constraints with change in some traits restricted to zero}

If constraints only include no change in some traits, then $K g$ is simpler and to form (9) one just deletes rows and columns from (3) corresponding to $\tilde{u}_{\mathrm{z}} \cdot \mathbf{K}_{0}$ is $q \times(q-z)$ can then be taken as

$$
\mathbf{K}_{0}^{\prime}=\left[\begin{array}{ll}
\mathbf{0} & \mathbf{I}_{\mathrm{N}}
\end{array}\right]^{\prime}
$$

and

$$
\tilde{\mathbf{u}}=\hat{\mathbf{u}}_{\mathrm{N}}
$$

\subsection{Constraints for desired changes}

If constraints are for proportional changes (predetermined relative changes) for all traits, then

$$
\mathbf{K}_{0}=\mathbf{k}
$$

and

$$
\tilde{\mathbf{u}}=\hat{\mathbf{u}}_{\mathbf{p}}
$$

The size of restricted BLUP equations corresponding to random additive effects is reduced to that of single trait BLUP.

\subsection{Animal model without repeated records}

If we denote the equations (9) as $\mathbf{W h}=\mathbf{h}$, an equivalent set of equations for $\mathbf{B}$ and $\tilde{\mathbf{u}}$ is

$$
\mathbf{Q W Q Q}^{-1} \hat{\mathbf{h}}=\mathbf{Q v}
$$

where

$$
\mathbf{Q}=\left[\begin{array}{ccc}
\mathbf{I}_{t} & \mathbf{0} & \mathbf{0} \\
\mathbf{0} & \mathbf{I}_{m(q-r)} & \mathbf{0} \\
\mathbf{0} & \mathbf{0} & \mathbf{I}_{r} \otimes \mathbf{A}^{-1}
\end{array}\right]
$$

where $t$ is the number of the columns of $\mathbf{X}$. Then because $\left(\mathbf{I}_{r} \otimes \mathbf{A}^{-1}\right) \mathbf{C}^{\prime} \mathbf{G}=$ $\mathbf{C}_{0}^{\prime} \mathbf{G}_{0} \otimes \mathbf{I}_{m}$ equations (9) are represented as in (10)

$$
\left[\begin{array}{lll}
\mathbf{X}^{\prime} \mathbf{R}^{-1} \mathbf{X} & \mathbf{X}^{\prime} \mathbf{R}^{-1} \mathbf{Z K} & \mathbf{X}^{\prime} \mathbf{R}^{-1} \mathbf{Z P} \\
\mathbf{K}^{\prime} \mathbf{Z}^{\prime} \mathbf{R}^{-1} \mathbf{X} & \mathbf{K}^{\prime} \mathbf{Z}^{\prime} \mathbf{R}^{\prime}-1 \mathbf{Z K}+\mathbf{K}^{\prime} \mathbf{G}^{-1} \mathbf{K} & \mathbf{K}^{\prime} \mathbf{Z}^{\prime} \mathbf{R}^{-1} \mathbf{Z} \mathbf{P} \\
\mathbf{P}^{\prime} \mathbf{Z}^{\prime} \mathbf{R}^{-1} \mathbf{X} & \mathbf{P}^{\prime} \mathbf{Z}^{\prime} \mathbf{R}^{-1} \mathbf{Z K} & \mathbf{P}^{\prime} \mathbf{Z}^{\prime} \mathbf{R}^{-1} \mathbf{Z P}
\end{array}\right]\left[\begin{array}{c}
\mathbf{6} \\
\tilde{\mathbf{u}} \\
\hat{\mathbf{s}}
\end{array}\right]=\left[\begin{array}{l}
\mathbf{X}^{\prime} \mathbf{R}^{-1} \mathbf{y} \\
\mathbf{K}^{\prime} \mathbf{Z}^{\prime} \mathbf{R}^{-1} \mathbf{y} \\
\mathbf{P}^{\prime} \mathbf{Z}^{\prime} \mathbf{R}^{-1} \mathbf{y}
\end{array}\right]
$$


where $\mathbf{p}^{\prime}=\mathbf{C}_{0}^{\prime} \mathbf{G}_{0} \otimes \mathbf{I}_{m}$ and $\hat{\mathbf{s}}=\left(\mathbf{I}_{r} \otimes \mathbf{A}^{-1}\right) \hat{\mathbf{w}}$ (see Quaas and Henderson [13]). If $\mathbf{C}=\mathbf{C}_{0} \otimes \mathbf{I}_{m}$, then we have $\mathbf{Z} \mathbf{Z}^{\prime}=\mathbf{I}$ and eliminating $\hat{\mathbf{s}}$ from (10), we obtain

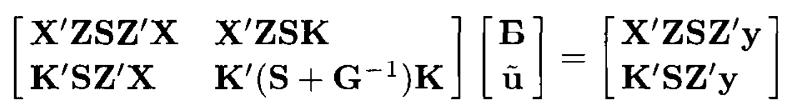

where $\mathbf{S}=\mathbf{Z}^{\prime} \mathbf{R}^{-1} \mathbf{Z}-\mathbf{Z}^{\prime} \mathbf{R}^{-1} \mathbf{Z P}\left(\mathbf{P}^{\prime} \mathbf{Z}^{\prime} \mathbf{R}^{-1} \mathbf{Z P}\right)-\mathbf{P}^{\prime} \mathbf{Z}^{\prime} \mathbf{R}^{-1} \mathbf{Z}[13]$ and $\mathbf{Z}$ is rows of $\mathbf{I}$ corresponding to missing records are deleted if there are missing records. Matrix $\mathbf{S}$ has simple forms and the calculation of their elements is easy. For example, $\mathbf{S}$ has the form

$$
\mathbf{S}=\left[\begin{array}{cccc}
\mathbf{D}_{11} & \mathbf{D}_{12} & \ldots & \mathbf{D}_{1 q} \\
\mathbf{D}_{12} & \mathbf{D}_{22} & \ldots & \mathbf{D}_{2 q} \\
\vdots & \vdots & & \vdots \\
\mathbf{D}_{1 q} & \mathbf{D}_{2 q} & \ldots & \mathbf{D}_{q q}
\end{array}\right]
$$

where each $\mathbf{D}_{i j}$ is a $m \times m$ diagonal matrix. Suppose that $d_{i j k}$ is the $k$ th element of $\mathbf{D}_{i j}$, then $d_{i j k}$ is the $i j$ th element of $\mathbf{S}_{k}$, that is, a matrix peculiar to the $k$ th animal, and

$$
\mathbf{S}_{k}=\mathbf{H}_{k}-\mathbf{H}_{k} \mathbf{G}_{0} \mathbf{C}_{0}\left(\mathbf{C}_{0}^{\prime} \mathbf{G}_{0} \mathbf{H}_{k} \mathbf{G}_{0} \mathbf{C}_{0}\right)-\mathbf{C}_{0}^{\prime} \mathbf{G}_{0} \mathbf{H}_{k}
$$

where $\mathbf{H}_{k}$ is a $q \times q$ matrix peculiar to the $k$ th animal. As shown by Quaas and Henderson [13], it is computed as follows:

1) if the animal has no records missing, $\mathbf{H}_{k}=\mathbf{R}_{0}^{-1}$, where $\mathbf{R}_{0}$ is the $q \times q$ error variance-covariance matrix;

2) if the animal has all records missing, $\mathbf{H}_{k}=\mathbf{0}$;

3) otherwise, find the inverse of the elements of $\mathbf{R}_{0}$ pertaining to records that are present and fill out the remaining elements with zeros for the other elements of $\mathbf{H}_{k}$.

\section{NUMERICAL EXAMPLE}

A numerical example obtained from the study of Henderson and Quaas [6] is used to illustrate the method. Data on five animals for birth weight (BW), weaning weight (WW) and feedlot gain (FG) are used and are as follows:

\begin{tabular}{lcccc}
\hline Animal & $\begin{array}{c}\text { Season } \\
\text { of birth }\end{array}$ & BW & WW & FG \\
\hline 1 & 1 & 61 & 362 & 1.96 \\
2 & 1 & 72 & 401 & 2.05 \\
3 & 2 & 68 & 350 & 1.81 \\
4 & 2 & 78 & 410 & 2.01 \\
5 & 2 & 65 & 340 & 1.74 \\
\hline
\end{tabular}


The genetic and error variance-covariance matrices are

and

$$
\begin{gathered}
\mathbf{G}_{0}=\left[\begin{array}{ccl}
28.60 & 73.77 & 0.50 \\
73.77 & 566.0 & 2.29 \\
0.50 & 2.29 & 0.0276
\end{array}\right] \\
\mathbf{R}_{0}=\left[\begin{array}{ccc}
36.3 & 67.43 & 0.06 \\
67.43 & 1454.0 & -0.53 \\
0.06 & -0.53 & 0.0254
\end{array}\right]
\end{gathered}
$$

respectively. The fixed effects in the model are a common mean for BW, and season of birth for WW and FG. Consequently,

$$
\mathbf{X}_{1}^{\prime}=\left[\begin{array}{lllll}
1 & 1 & 1 & 1 & 1
\end{array}\right]
$$

and

$$
\mathbf{X}_{2}^{\prime}=\mathbf{X}_{3}^{\prime}=\left[\begin{array}{lllll}
1 & 1 & 0 & 0 & 0 \\
0 & 0 & 1 & 1 & 1
\end{array}\right]
$$

Because all animals are assumed to have all records for the three traits, then

$$
\mathbf{Z}_{1}=\mathbf{Z}_{2}=\mathbf{Z}_{3}=\mathbf{I}_{5}
$$

The additive genetic relationship matrix is

$$
\mathbf{A}=\left[\begin{array}{llllc}
1.0 & 0.25 & 0.25 & 0.0 & 0.0 \\
0.25 & 1.0 & 0.25 & 0.0 & 0.0 \\
0.25 & 0.25 & 1.0 & 0.0 & 0.0 \\
0.0 & 0.0 & 0.0 & 1.0 & 0.25 \\
0.0 & 0.0 & 0.0 & 0.25 & 1.0
\end{array}\right]
$$

Suppose that the restrictions are for no genetic change in BW and for desired changes in WW and FG which are one genetic standard deviation unit, namely 23.79:0.1661. Then

$$
\mathbf{C}_{0}^{\prime}=\left[\begin{array}{llr}
0.1661 & 0.0 & 0.0 \\
0.0 & 0.1661 & -23.79
\end{array}\right]
$$

First, the direct solution of restricted BLUP will be shown. Matrices $\mathbf{X}^{\prime} \mathbf{R}^{-1} \mathbf{X}$, $\mathbf{X}^{\prime} \mathbf{R}^{-1} \mathbf{Z}, \mathbf{X}^{\prime} \mathbf{R}^{-1} \mathbf{Z G C}, \mathbf{Z}^{\prime} \mathbf{R}^{-1} \mathbf{Z}+\mathbf{G}^{-1}, \mathbf{Z}^{\prime} \mathbf{R}^{-1} \mathbf{Z G C}$ and $\mathbf{C}^{\prime} \mathbf{G} \mathbf{Z}^{\prime} \mathbf{R}^{-1} \mathbf{Z G C}$ of equations (3) become (12a), (12b), (12c), (12d), (12e) and (12f), respectively.

$$
\mathbf{X}^{\prime} \mathbf{R}^{-1} \mathbf{X}=\left[\begin{array}{rrrrr}
0.1520 & -.0029 & -.0043 & -.2040 & -.3061 \\
-.0029 & 0.0015 & 0.0000 & 0.0386 & 0.0000 \\
-.0043 & 0.0000 & 0.0023 & 0.0000 & 0.0580 \\
-.2040 & 0.0386 & 0.0000 & 80.0283 & 0.0000 \\
-.3061 & 0.0000 & 0.0580 & 0.0000 & 120.0424
\end{array}\right]
$$


M. Satoh

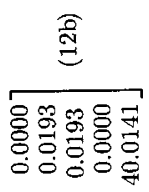

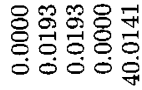

응영융형

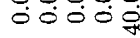

ఇ융응ㅋㅇㅇ

000 尚

ஓ융융평형 ○ं 0 宊

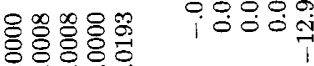

영ㅇㅇ

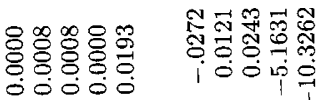

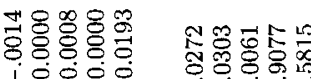

iơ

평융융ㅎㅀ융

io:00

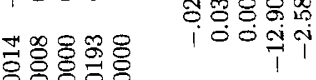

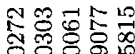

io0.0

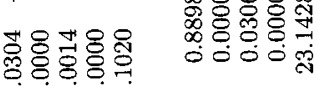
0 Oioi

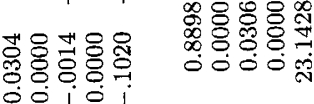

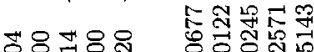

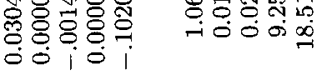

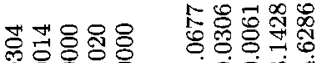
- 0 ○ु O0ं०

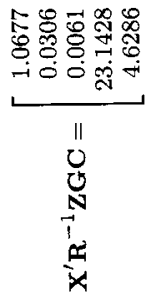

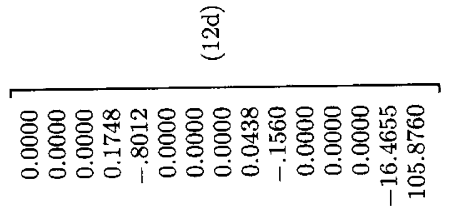

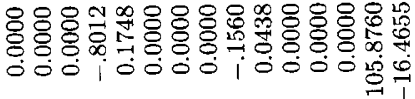

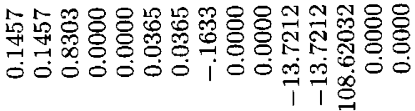

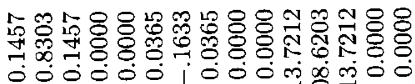

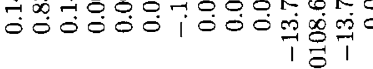

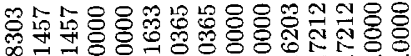
○

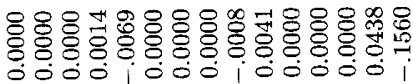

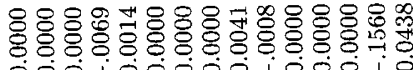

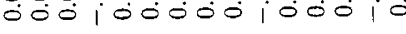

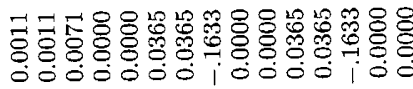

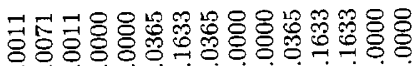
ii

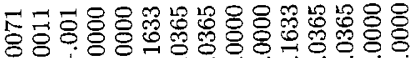
191850908090800

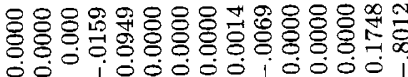

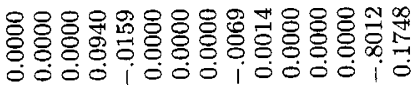

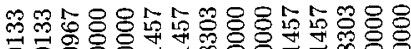
i i

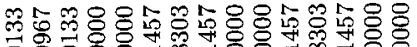

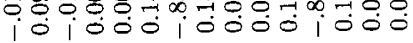
흐으. Sil0010000000 


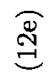

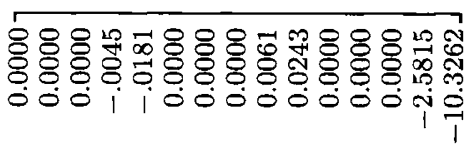

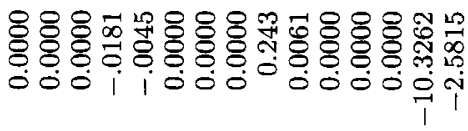

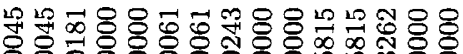

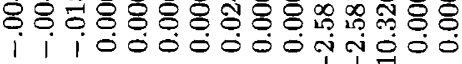
i 1

늄 8ิว

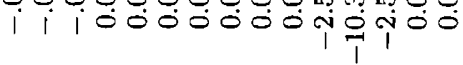

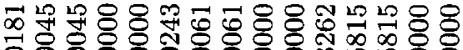

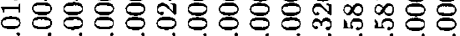

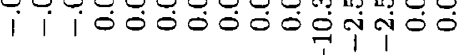

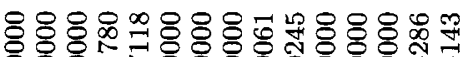

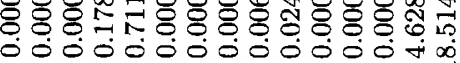

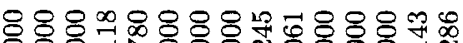
ㅇㅇㅇㅇㅇㅇ첟ㅇㅇㅇㅇㅇㅇㅇㅇㅇㅇㅇㅇㅇㅇㅇำ

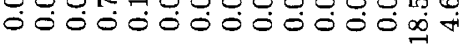

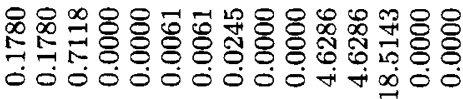

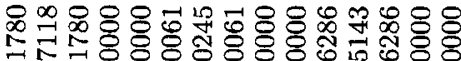

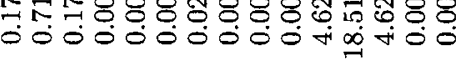

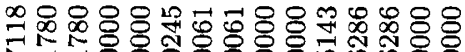

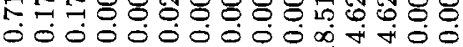

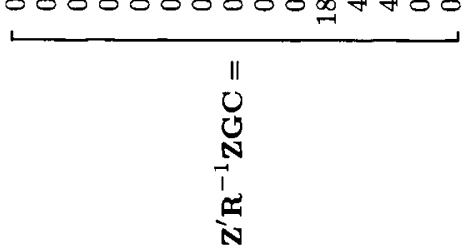

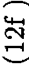

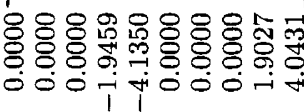

용유융용요 ठㅇㅇㅇㅇㅠ

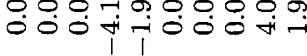

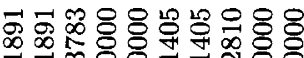
开

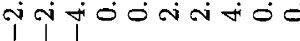

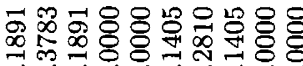
i

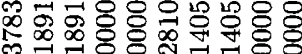
i

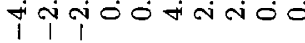

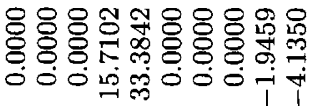

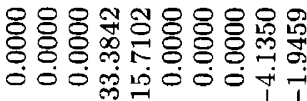

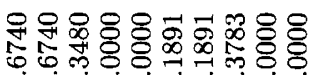

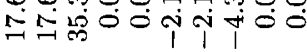

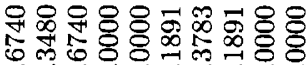
出

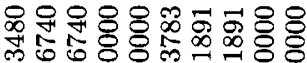

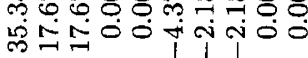

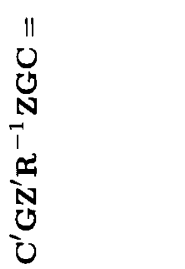


Vectors $\mathbf{X}^{\prime} \mathbf{R}^{-1} \mathbf{y}, \mathbf{Z}^{\prime} \mathbf{R}^{-1} \mathbf{Z}$ and $\mathbf{C}^{\prime} \mathbf{G} \mathbf{Z}^{\prime} \mathbf{R}^{-1} \mathbf{y}$ of equations (3) are $\mathbf{X}^{\prime} \mathbf{R}^{-1} \mathbf{y}=\left[\begin{array}{llllll}6.7868 & 0.4663 & 0.6401 & 161.6266 & 22.2007\end{array}\right]^{\prime}$,

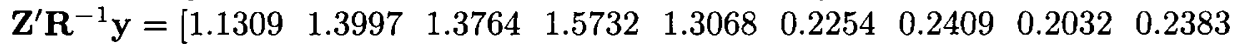

$\left.\begin{array}{llllll}0.1986 & 79.1971 & 82.4295 & 72.2491 & 80.3907 & 69.5610\end{array}\right]^{\prime}$, $\mathbf{C}^{\prime} \mathbf{G} \mathbf{Z}^{\prime} \mathbf{R}^{-1} \mathbf{y}=\left[\begin{array}{llllll}143.7809 & 137.3768 & 124.4704 & 112.4962 & -18.6050 & -18.7419\end{array}\right.$ $-17.7570-14.9453-13.9514]^{\prime}$.

Because rank $\left(\mathbf{X}^{\prime} \mathbf{Z} \mathbf{S Z} \mathbf{Z}^{\prime} \mathbf{X}\right)=2$, solving the equations requires finding a generalized inverse of (4) which may be obtained by setting appropriate columns of $\mathbf{Z}^{\prime} \mathbf{X}$ to $\mathbf{0}$ to remove all linear dependencies and then inverting the non-null portion. For this example, $\mathbf{X}_{1}$ and $\mathbf{X}_{2}$ are assumed to be $\mathbf{0}$. From these equations, solutions are as shown in table $I$.

Next, the technique developed here will be shown. From (9),

$$
\mathbf{K}^{\prime}=\mathbf{K}_{0}^{\prime} \otimes \mathbf{I}_{5}=\left[\begin{array}{lll}
0.0 & 143.2035 & 1.0
\end{array}\right] \otimes \mathbf{I}_{5}=\left[\begin{array}{lll}
0.0 \otimes \mathbf{I}_{5} & 143.2035 \otimes \mathbf{I}_{5} & 1.0 \otimes \mathbf{I}_{5}
\end{array}\right],
$$

then matrices $\mathbf{X}^{\prime} \mathbf{R}^{-1} \mathbf{Z K}, \quad \mathbf{K}^{\prime} \mathbf{Z}^{\prime} \mathbf{R}^{-1} \mathbf{Z K}+\mathbf{K}^{\prime} \mathbf{G}^{-1} \mathbf{K}$ and $\mathbf{K}^{\prime} \mathbf{Z}^{\prime} \mathbf{R}^{-1} \mathbf{Z G C}$ of equations [9] become (12a), (12b) and (12c), respectively.

$$
\mathbf{X}^{\prime} \mathbf{R}^{-1} \mathbf{Z K}=\left[\begin{array}{rrrrr}
-0.3093 & 0.3093 & -0.3093 & -0.3093 & -0.3093 \\
0.1284 & 0.1284 & 0.0000 & 0.0000 & 0.0000 \\
0.0000 & 0.0000 & 0.1284 & 0.1284 & 0.1284 \\
42.7804 & 42.7804 & 0.0000 & 0.0000 & 0.0000 \\
0.0000 & 0.0000 & 42.7804 & 42.7804 & 42.7804
\end{array}\right]
$$

$$
\begin{aligned}
\mathbf{K}^{\prime} \mathbf{Z}^{\prime} \mathbf{R}^{-1} \mathbf{Z K}+\mathbf{K}^{\prime} \mathbf{G}^{-1} \mathbf{K}= \\
{\left[\begin{array}{rrrrr}
148.0820 & -17.3821 & -17.3821 & 0.0000 & 0.0000 \\
-17.3821 & 148.0820 & -17.3821 & 0.0000 & 0.0000 \\
-17.3821 & -17.3821 & 148.0820 & 0.0000 & 0.0000 \\
0.0000 & 0.0000 & 0.0000 & 144.6056 & -20.8585 \\
0.0000 & 0.0000 & 0.0000 & -20.8585 & 144.6056
\end{array}\right] }
\end{aligned}
$$

and

$$
\mathbf{K}^{\prime} \mathbf{Z}^{\prime} \mathbf{R}^{-1} \mathbf{Z G C}=\left[\begin{array}{rcccc}
22.0190 & 5.5047 & 5.5047 & 0.0000 & 0.0000 \\
5.5047 & 22.0190 & 5.5047 & 0.0000 & 0.0000 \\
5.5047 & 5.5047 & 22.0190 & 0.0000 & 0.0000 \\
0.0000 & 0.0000 & 0.0000 & 22.0190 & 5.5047 \\
0.0000 & 0.0000 & 0.0000 & 5.5047 & 22.0190 \\
-6.8511 & -1.7128 & -1.7128 & 0.0000 & 0.0000 \\
-1.7128 & -6.8511 & -1.7128 & 0.0000 & 0.0000 \\
-1.7128 & -1.7128 & -6.8511 & 0.0000 & 0.0000 \\
0.0000 & 0.0000 & 0.0000 & -6.8511 & -1.7128 \\
0.0000 & 0.0000 & 0.0000 & -1.7128 & -6.8511
\end{array}\right]
$$


The solutions for $\mathrm{FG}$ give the predicted breeding values which are identical to table I. From (6) and (7), $\hat{\mathbf{u}}$ of $\mathrm{BW}=\mathbf{0}$ and $\hat{u}$ of $\mathrm{WW}=143.227 \times \hat{\mathbf{u}}$ of FG., which are identical to table 1 .

Table I. Solutions of each animal.

\begin{tabular}{lccr}
\hline Animal & BW & WW & FG \\
\hline 1 & 0.000 & -0.2227 & -0.0016 \\
2 & 0.000 & -0.708 & -0.0049 \\
3 & 0.000 & -1.870 & -0.0131 \\
4 & 0.000 & 4.203 & 0.0293 \\
5 & 0.000 & -1.866 & 0.0130 \\
\hline
\end{tabular}

In this example, equations [11] are useful.

$$
\mathbf{S}=\mathbf{S}_{0} \otimes \mathbf{I}_{m}=\left[\begin{array}{rrr}
0.0128 & -0.0026 & -0.3522 \\
-0.0026 & 0.0005 & 0.0709 \\
-0.3522 & 0.0709 & 9.6875
\end{array}\right] \otimes \mathbf{I}_{5},
$$

then, the matrix on the left hand side of equations (11) presented in (14) were obtained by removing rows and columns of all linear dependencies.

$\left[\begin{array}{rrrrrrr}19.3749 & 0.0000 & 19.8395 & 19.8395 & 0.0000 & 0.0000 & 0.0000 \\ 0.0000 & 29.0624 & 0.0000 & 0.0000 & 19.8395 & 19.8395 & 19.8395 \\ 19.8395 & 0.0000 & 127.5556 & -17.3850 & -17.3850 & 0.0000 & 0.0000 \\ 19.8395 & 0.0000 & -17.3850 & 127.5556 & -17.3850 & 0.0000 & 0.0000 \\ 0.0000 & 19.8395 & -17.3850 & -17.3850 & 127.5556 & 0.0000 & 0.0000 \\ 0.0000 & 19.8395 & 0.0000 & 0.0000 & 0.0000 & 124.0786 & -20.8620 \\ 0.0000 & 19.8395 & 0.0000 & 0.0000 & 0.0000 & -20.8620 & 124.0786\end{array}\right]$

We also obtain the expected breeding values which are identical to (13) from the solutions using (14).

\section{DISCUSSION}

Henderson and Quaas [6] derived BLUP of breeding values for multiple traits using records on a large number of relatives. Restricted BLUP was derived by imposing restrictions on multiple trait BLUP [13]. Hence, in restricted BLUP, the computing load to obtain estimates of breeding values can be huge.

Itoh and Iwaisaki [7] showed that a canonical transformation technique was applicable to restricted BLUP in order to reduce the number of equations for 
an animal model. However, the method has a limitation in that models must be identical for all traits and there must be no partially missing observations. Hence, the canonical transformation technique can be used only if models and data structure conform to the above conditions.

Various restricted selection index theories have been presented since Kempthorne and Nordskog [9]. However, only two types of constraints have been used practically for selection, i.e. zero changes in one or a few traits and proportional changes for all traits (e.g. Hagger [2]). Now if all constraints are zero-change for some traits and $m$ is several millions even removing a $\hat{\mathbf{u}}$ equation for a set of constraints might be useful. If proportional changes are imposed for all traits, the size of equations corresponding to random additive genetic effects is much reduced. The technique developed here needs no conditions to be applied and reduced the number of sets of equations corresponding to random additive effects from $q$ to $q$-rank $\left(\mathbf{C}_{0}\right)$. Hence, if a large number of restrictions is imposed in a model such as a constraint of achieving predetermined relative changes for all traits $[1,12,16]$, the size of equations for random additive effects is the same as that of a single trait model.

\section{REFERENCES}

[1] Brascamp E.W., Selection indices with constraints, Anim. Breed. Abstr. 52 (1984) $645-654$.

[2] Hagger C., Two generations of selection on restricted best linear unbiased prediction breeding values for income minus feed cost in laying hens, J. Anim. Sci. 70 (1992) 20452052

[3] Harville D.A., Optimal procedures for some constrained selection index problems, J. Am. Stat. Assoc. 69 (1974) 446-452.

[4] Harville D.A., Index selection with proportionality constraints, Biometrics 31 (1975) 223-225.

[5] Henderson C.R., Sire evaluation and genetic trends, in: Proceedings of the Animal Breeding and Genetics Symposium in honor of Dr JL Lush, Blacksburg, VA, August 1973, American Society of Animal Science, Champaign, IL, 1973, pp. 10-41.

[6] Henderson C.R., Quaas R.L., Multiple trait evaluation using relatives' records, J. Anim. Sci. 43 (1976) 1188-1197.

[7] Itoh Y., Iwaisaki H., Restricted best linear unbiased prediction using canonical transformation, Genet. Sel. Evol. 22 (1990) 339-347.

[8] Itoh Y., Yamada Y., Comparisons of selection indices achieving predetermined proportional gains, Genet Sel. Evol. 19 (1987) 69-82.

[9] Kempthorne O., Nordskog A.W., Restricted selection indices, Biometrics 15 (1959) $10-19$.

[10] Lin C.Y., A unified procedure of computing restricted best linear unbiased prediction and restricted selection index, J. Anim. Breed. Genet. 107 (1990) 1-316.

[11] Mallard J., The theory and computation of selection indices with constraints: a critical synthesis, Biometrics 28 (1972) 713-735.

[12] Pesek J., Baker R.J., Desired improvement in relation to selection indices, Can. J. Plant. Sci. 49 (1969) 803-804. 
[13] Quaas R.L., Henderson C.R., Restricted best linear unbiased prediction of breeding values, Mimeo, Cornell Univ., Ithaca, NY, 1976, pp. 1-14.

[14] Quaas R.L., Henderson C.R., Selection criteria for altering the growth curve, J. Anim. Sci. (abstr) 43 (1976) 221.

[15] Schneeberger M., Barwick S.A., Crow G.H., Hammond K., Economic indices using breeding values predicted by BLUP, J. Anim. Breed. Genet. 109 (1992) 180-187.

[16] Yamada Y, Yokouchi K., Nishida A., Selection index when genetic gains of individual traits are of primary concern, Jpn. J. Genet. 50 (1975) 33-41. 